\title{
A Survey on Web OS
}

\author{
Ginu Jose ${ }^{1}$, Reshma Sebastian ${ }^{2}$, Deepa Merin Jose ${ }^{3}$ \\ Student, Department of CSE, MBC College of Engineering \& Technology, Peermade, India ${ }^{1,2}$ \\ Assistant Professor, Department of CSE, MBC College of Engineering \& Technology, Peermade, India ${ }^{3}$
}

\begin{abstract}
Cloud used as a standalone operating system, where the hardware required is relatively low. Cloud OS manages the user account and the Cloud OS include simple Text editor, Paint application, spreadsheet and a presentation viewer that we usually use in daily life. It also includes Calendar, Contacts manager, and simple games. The Web OS is also used for Remote application management. An OS provide an interface for user's to manage the applications running on the system. Cloud computing is the technology that helps in reducing the usage of time, cost and resources which is now-a-days used widely by the companies. There are several cloud approaches to store data files and synchronize file versions.
\end{abstract}

Keywords: Cloud OS, GUI Interface, Chrome OS, Virtual Desktop, SaaS, PaaS, IaaS.

\section{INTRODUCTION}

Cloud OS is a simple operating system that runs just a web browser that provides access to a variety of web-based applications that allow the user to perform many simple tasks without booting a full-scale operating system. Because of its simple structure, Cloud OS can boot in just a few seconds. The operating system is designed for Mobiles as well as PC's. The cloud has three forms of models: Software as a Service (SaaS), Platform as a Service (PaaS), and Infrastructure as a Service (IaaS). The cloud web desktop (Cloud Me) is also a Web OS provided that it offers a virtual desktop for its clients, however it cannot be offered through the web.

\section{THE USE OF WEB OS}

A Web Operating System or an Online Operating System is a user interface that allows users to access a desktop and applications installed on the Web. It is considered being similar to the user interface of traditional computer operating systems like Windows or Linux but it does not directly interact with the computer's hardware resources or the system architecture[5].

Our primary objective is to develop a Web based OS with core functionalities that can be integrated with social media. The secondary objective includes a provision to provide VOIP service for cloud OS users. We know that, there are many operating system available on web. The commonly available Web OS are: XML based lucid web operating system, Cloud OS, SEWOS, Virtual remote web desktop, Megha OS. The cloud web OS has a virtual desktop that let the users access their files and photos in folders and icons such as most of the regular OS desktops, while, most of the other cloud applications don't have virtual desktop.

It is easy to develop and add any feature in future to this system. So any service or application that needed to be added in future, can be developed alone then can be added to the cloud web OS. Some of the advantages of existing operating systems are: We can access our desktop from anywhere, where it supports client devices. No need to change multiple clients. Administrator has the responsibility and resources to control the application and the users can access the data from anywhere. The OS can be accessed irrespective of hardware limitations of the system used. They ensure all users are running the updated versions of all applications.

Web OS is a new form of operating system in which the virtual desktop on the web is accessible by browsers with multiple integrated built in applications that allow user to easily manage and organize the data from any location. Desktop on the web can be termed as WEBtop[6].

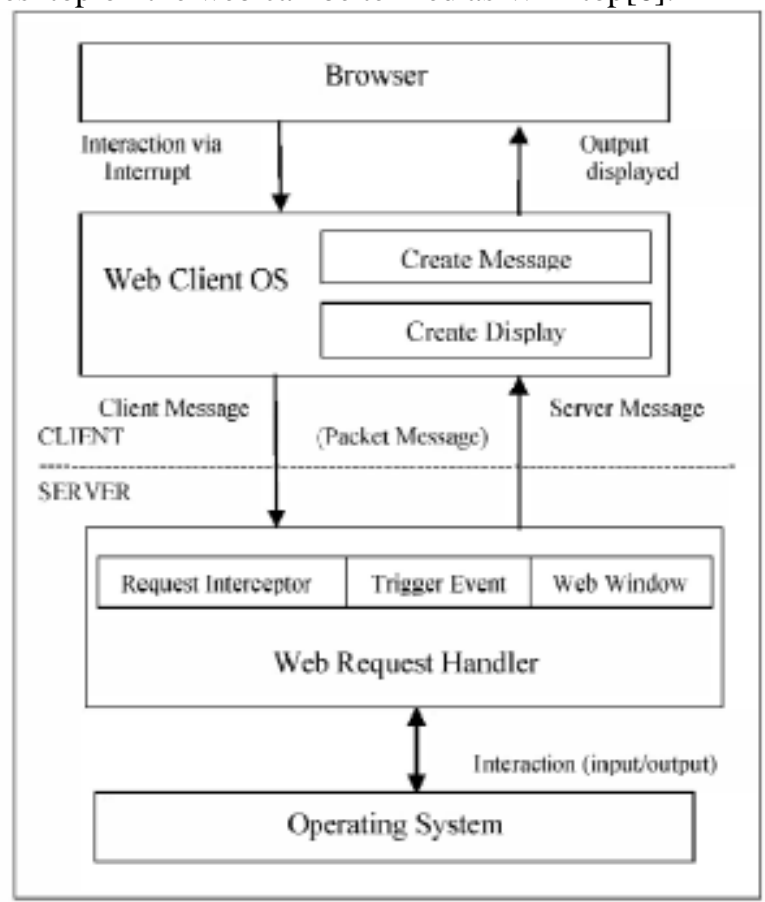

Fig 1. Basic architecture of Virtualized Remote Web Desktop

\section{A. User Interface}

User interface should be of the form that uses the minimal space by combining applications and standards into Web pages of single tab strip, rather than separating the two. Secondary tasks are handled by "panels', floating windows at the bottom of the screen for chat and music players. There are many cloud services that serves as a wide range from business use to social use and from 
learning purposes to news and media purposes, but the When we look at the XML based Lucid Web OS, the main problem with these services are that they are scattered features are:

which makes the user distracted by many pages and many

GUIs in such way that it is not time efficient for hard core 1. OS will not store any application on device itself. web users[2].

2. Support for both $\mathrm{x} 86$ and ARM architecture

\section{B. $\quad$ Cloud Computing}

3. Smooth execution of VM.

The cloud in cloud computing is a set of hardware and 5. Management of VM images.

software that combine to deliver aspects of computing as a 6 . Dynamic creation and movement of VM and service.

associated storage.

There are many types of cloud computing that provide 7. Definition of security policies on the users of the various kinds of service areas that are classified into 4:

Public Cloud, Private Cloud, Community Cloud, Hybrid Cloud.

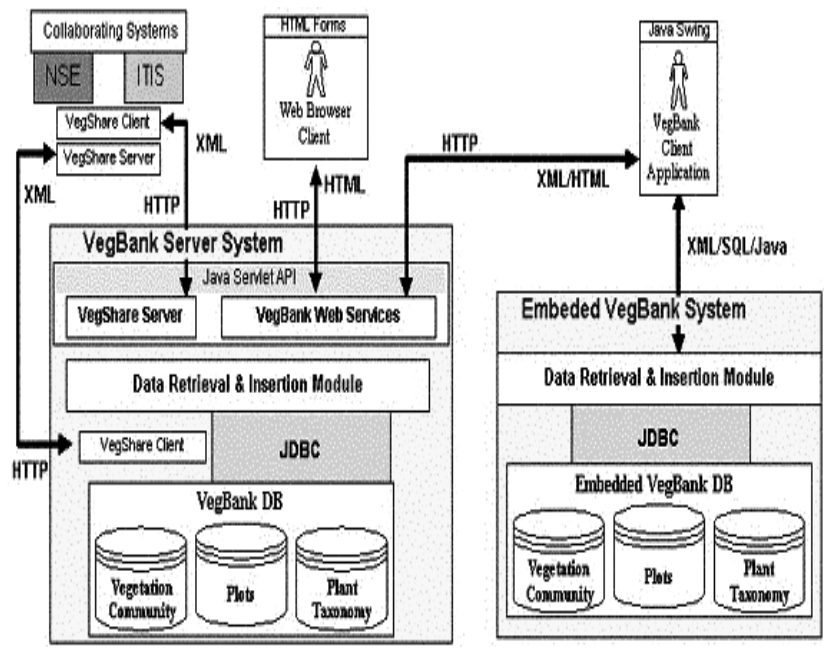

Fig 2. Framework of Web based Operating System

\section{C. $\quad$ The Underlying Technologies}

The Cloud OS has a Web Desktop, which has a GUI that is capable of containing multiple windows and that allow users to make their normal cloud activities with friendly interfaces. The user side of Web OS software, that can run through a Web browser or a system-specific client, runs on top of the computer's OS.

While programming the software, the system ultimately has to act like a desktop OS and its look and feel retained. 1 . Normally a Web OS might look a lot like a traditional OS, 2. but it does not resemble the computer's hardware or 3 . software. While the goal of a Web OS is to provide a 4 . feeling which is similar to in using a desktop OS, there are 5 . no rules for how to make this happen. The two most 6 . popular strategy now we employ is the Flash technologies 7 . or Asynchronous JavaScript and XML (AJAX) technologies [5].

\section{III.THE TRENDS OF EXISTING OPERATING SYSTEM}

The existing operating system has several features which have several advantages and disadvantages. With the existing operating system, it is found that there are some limitations, so that we have designs for a new Web OS with similar as well as new features. The existing Web OS can be used from anywhere with a browser. It provides the simplicity in usage as well user friendly interface. system.

8. Management of Remote Cloud Capacity.

Some other similar characteristics of Cloud OS when compared with other Web OS are:

1. The cloud web OS has a virtual desktop that let the users access their files and photos in folders and icons.

2. The cloud web OS can be accessed from any web browser on PC, Mac, and Linux and also can be accessed from any Mobile device has a web browser.

3. It is easy to develop and add any feature in future to our system.

4. There is no need to pay license for any product since it's available or we have similar applications in cloud server.

5. The cloud server can also act as router in the internal network.

Cloud web operating system includes some features:

1. Access to Cloud-wide object and process naming via DNS and/or other distributed naming services

2. Distributed reliable storage functionality

3. Automated Cloud application deployment, horizontal scaling, and lifecycle management

4. High availability failover support with check pointed replicated process execution.

Wed Desktop includes some additional features:

Software and hardware management.

Constant API.

Interruption.

Execution of programs.

Managing memory

Networking.

Security.

The main components that are included in this system are: Browser, Web client OS, Web request handler.

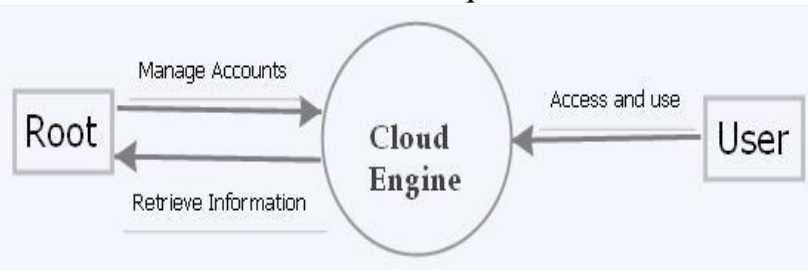

Fig 3. The typical diagram of Cloud and its users 


\section{IV.CONCLUSION}

Cloud OS aims to provide an expensive set of resource management options and metrics of applications to facilitate programming in cloud. The use of commands make load lighter in the network providing the GUI interface. This can lead to better performance in service. There are many cloud applications and web OS that are used in various fields. Most of these applications are used to synchronise files and photos on different device using a third party device which is the cloud server. This cloud web OS can be also used by developer to compile and run Java and $\mathrm{C}++$ applications. It can be also used to format documents, sheets and office applications files. Social web account can be integrated on the cloud web OS to simplify the access of the social media web sites. It also provides facility to access from any web browser on any device.

\section{REFERENCES}

[1] Kapil Garg, Ankit Agarwal, Mayur Gaikward, Vahid Inamdar, Arvind Rajpurohit - "XML based Lucid Web Operating System", 2008.

[2] Hesham Abusaimeh - "Cloud Web based operating system", International Journal of Computer Science \& Information Technology (IJCSIT) Vol 6, No 2, April 2014, pp. 41- 48.

[3] Sanil C.Savale -"Trends in Cloud Operating System", International Conference on Recent Trends in Information Technology and Computer Science (IRCTITCS) 2011 Proceedings published in International Journal of Computer Applications® (IJCA).

[4] Amina Sultana, Bittu Daimary, Mahesh Chettri, Joby Joseph "Virtualized Remote Web Operating System", pp. 1- 4, 2012.

[5] Ananya Tripathi - "Web Operating System", Open Access Library Journal, 2014, 1, 1-5, pp. 2- 4.

[6] Dr. M.K Sharma and Rajeev Kumar - "WEBtop(Operating Systems on Web)" 